\title{
Б.О. Цыденов
}

\section{МАТЕМАТИЧЕСКАЯ МОДЕЛЬ ДЛЯ ВОСПРОИЗВЕДЕНИЯ БИОГЕОХИМИЧЕСКИХ ПРОЦЕССОВ В ПРЕСНОВОДНОМ ОЗЕРЕ}

\begin{abstract}
Предложена математическая модель для исследования экологического состояния пресноводного озера, которая включает в себя 10 прогностических переменных: нитрат, фосфат, аммоний, хлорофилл $a$, фитопланктон, зоопланктон, а также нитратный и фосфатный детрит мелких и крупных размеров. Численно воспроизведены биогеохимические процессы в Баргузинском заливе оз. Байкал в летний период. Проведено сопоставление полученных результатов с данными натурных измерений.
\end{abstract}

Ключевые слова: математическая модель, экосистема пресноводного озера, численный эксперимент, озеро Байкал.

В последние десятилетия под влиянием деятельности человека происходит заметное ухудшение экологического состояния крупных пресноводных озер планеты. Большой вклад в загрязнение и эвтрофирование водоемов вносят промышленные центры, сосредоточенные на их побережье и/или территории водосборного бассейна. В частности, в литоральной зоне озера Байкал в последние годы наблюдается активный рост водорослей рода спирогира, который связан с городскими бытовыми и промышленными стоками с высоким содержанием азота и фосфора.

Важнейшими химическими элементами в озерной экосистеме выступают фосфор и азот, поскольку один из них является лимитирующим фактором развития биомассы водоема. Уровень концентрации фосфора в пресном озере имеет критически важное значение в предсказании вспышки цветения фитопланктона. В связи с этим проблема создания интегративных моделей, направленных на численное воспроизведение биохимических процессов фосфорного и азотного лимитирования, является актуальной для современного этапа развития математических методов в задачах лимнологии.

Несмотря на длительную историю изучения первичной продукции в озере Байкал, вопрос о лимитирующих факторах развития его биомассы остается открытым. К примеру, ряд ученых $[1,2]$ считает, что развитие фитопланктона в Байкале лимитируется азотом. Но в то же время Голдман и др. [3] отмечают, что в летние периоды более отчетливым становится значение как фосфорного, так и азотного лимитирования. Экспериментальные исследования в Баргузинском заливе в конце августа 2002 г. [4] указывают на роль фосфора как первостепенного лимитирующего биогенного элемента, однако при поступлении фосфора в поверхностные воды лимитирующим становится азот.

Очевидно, что при моделировании биохимических процессов в пресноводных экосистемах важно рассматривать не только наличие азота в воде, но и учитывать содержание фосфора, поскольку фосфатные группы являются основными струк-

\footnotetext{
${ }^{1}$ Исследование выполнено при финансовой поддержке РФФИ в рамках научного проекта № 19-3160003.
} 
турными элементами нуклеиновых кислот в растительных клетках и участвуют в энергетическом обмене [5]. Целью данной работы является разработка и описание математической модели экосистемы пресноводного озера с учетом фосфорного и азотного лимитирования для воспроизведения в нем физико-биологических процессов, а также анализ и обсуждение результатов расчетов, проведенных для условий озера Байкал в летний период.

\section{Математическая модель}

Математическая модель для воспроизведения физико-биологических процессов в пресноводном озере состоит из термогидродинамического $[6,7]$ и экосистемного блоков. Экосистемный блок основан на моделях Фашама и др. [8], Феннель и др. [9], Хофмана и др. [10] и включает в себя 7 прогностических переменных: нитрат (1), аммоний (2), хлорофилл $a$ (4), фитопланктон (5), зоопланктон (6), мелкий (7) и крупный (8) нитратный детрит. Для описания фосфорного лимитирования в пресноводной среде дополнительно введены в модель 3 переменные согласно постановке Гана и др. [11]: фосфат (3), мелкий (9) и крупный (10) фосфатный детрит. Схематическая диаграмма, демонстрирующая связь между компонентами экосистемы, представлена на рис. 1. Интенсивность роста фитопланктона в модели определяется через модули расчета фосфорной и азотной составляющих согласно их роли в лимитировании биомассы водоема [4]. В качестве индикатора лимитирования часто используют молярное отношение содержания минерального азота $(\mathrm{N})$ к содержанию минерального фосфора (Р). Согласно Редфилду [12], при соотношении N:P < 16 первичная продукция фитопланктона лимитируется азотом, а при $\mathrm{N}: \mathrm{P}>16$ - фосфором.

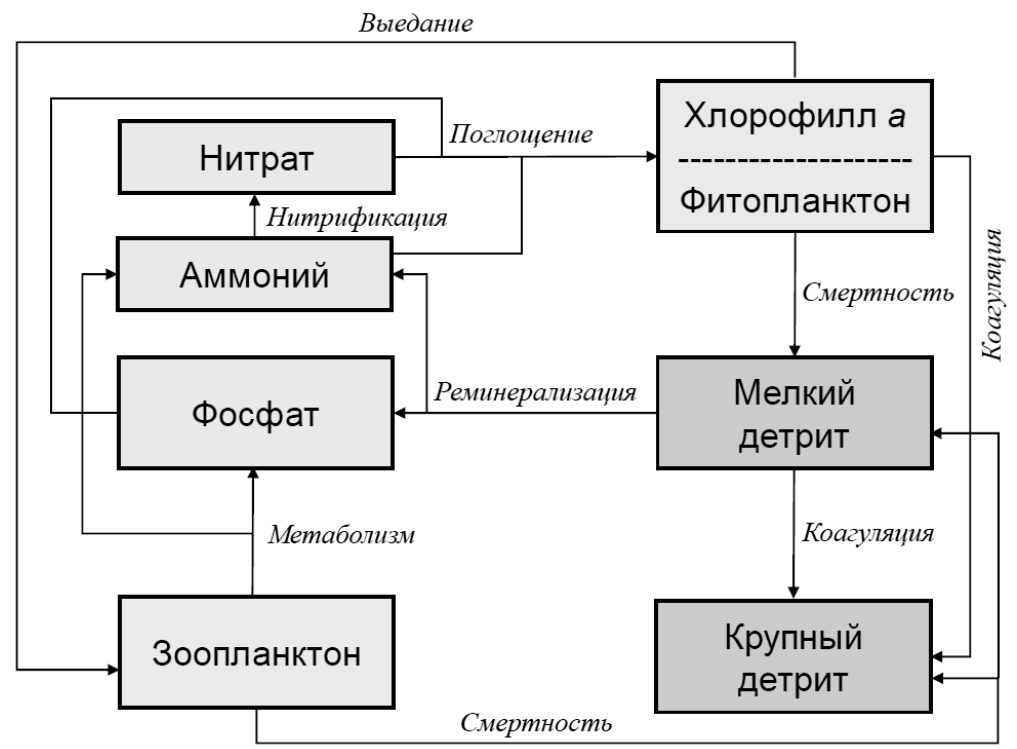

Рис. 1. Концептуальное представление экосистемного блока математической модели. Стрелками показаны биологические процессы между компонентами модели [11] Fig. 1. Conceptual representation of the ecosystem module of the mathematical model. Arrows indicate the biological processes between components of the model [11] 
Биохимические процессы в пресноводном озере описываются с помощью реакционно-конвективно-диффузионных уравнений

$$
\frac{\partial[N]}{\partial t}+\frac{\partial u[N]}{\partial x}+\frac{\partial w[N]}{\partial z}=\frac{\partial}{\partial x}\left(D_{x} \frac{\partial[N]}{\partial x}\right)+\frac{\partial}{\partial z}\left(D_{z} \frac{\partial[N]}{\partial z}\right)-
$$

$-\mu_{\max } f(I)[$ Phyto $]\left(\sigma_{N} \frac{[N]}{k_{N}+[N]} \cdot \frac{1}{1+[A] / k_{A}}+\sigma_{P} \frac{[N]}{[N]+[A]} \cdot \frac{[P]}{k_{P}+[P]}\right)+n[A] ;$

$$
\begin{gathered}
\frac{\partial[A]}{\partial t}+\frac{\partial u[A]}{\partial x}+\frac{\partial w[A]}{\partial z}=\frac{\partial}{\partial x}\left(D_{x} \frac{\partial[A]}{\partial x}\right)+\frac{\partial}{\partial z}\left(D_{z} \frac{\partial[A]}{\partial z}\right)- \\
-\mu_{\max } f(I)[\text { Phyto }]\left(\sigma_{N} \frac{[A]}{k_{A}+[A]}+\sigma_{P} \frac{[A]}{[N]+[A]} \cdot \frac{[P]}{k_{P}+[P]}\right)-
\end{gathered}
$$

$-n[A]+l_{B M}[$ Zoo $]+l_{E} \frac{[\text { Phyto }]^{2}}{k_{\text {Phyto }}+[\text { Phyto }]^{2}} \beta[Z o o]+r_{S D N}[S D N]+r_{L D N}[L D N]$;

$$
\frac{\partial[P]}{\partial t}+\frac{\partial u[P]}{\partial x}+\frac{\partial w[P]}{\partial z}=\frac{\partial}{\partial x}\left(D_{x} \frac{\partial[P]}{\partial x}\right)+\frac{\partial}{\partial z}\left(D_{z} \frac{\partial[P]}{\partial z}\right)-
$$

$-\mu_{\max } f(I)[$ Phyto $]\left(\sigma_{N}\left\{\frac{[N]}{k_{N}+[N]} \cdot \frac{1}{1+[A] / k_{A}}+\frac{[A]}{k_{N}+[A]}\right\} r_{P N}+\sigma_{P} \frac{[P]}{k_{P}+[P]} r_{P N}\right)+$

$$
+l_{B M} r_{P N}[\text { Zoo }]+l_{E} r_{P N} \frac{[P h y t o]^{2}}{k_{\text {Phyto }}+[\text { Phyto }]^{2}} \beta[Z o o]+r_{S D P}[S D P]+r_{L D P}[L D P] ;
$$

$\frac{\partial[C h l]}{\partial t}+\frac{\partial u[C h l]}{\partial x}+\frac{\partial w[C h l]}{\partial z}=\frac{\partial}{\partial x}\left(D_{x} \frac{\partial[C h l]}{\partial x}\right)+\frac{\partial}{\partial z}\left(D_{z} \frac{\partial[C h l]}{\partial z}\right)+\rho_{C h l} \mu[C h l]-$

$$
-m_{\text {Phyto }}[C h l]-\tau([S D N]+[\text { Phyto }])[C h l]-g_{\max } \frac{[\text { Phyto }]^{2}}{k_{\text {Phyto }}+[\text { Phyto }]^{2}}[\text { Zoo }] \frac{[C h l]}{[\text { Phyto }]} \text {; }
$$

$$
\frac{\partial[\text { Phyto }]}{\partial t}+\frac{\partial u[\text { Phyto }]}{\partial x}+\frac{\partial w[\text { Phyto }]}{\partial z}=\frac{\partial}{\partial x}\left(D_{x} \frac{\partial[\text { Phyto }]}{\partial x}\right)+\frac{\partial}{\partial z}\left(D_{z} \frac{\partial[\text { Phyto }]}{\partial z}\right)+
$$

$+\mu[$ Phyto $]-m_{\text {Phyto }}[$ Phyto $]-\tau([S D N]+[$ Phyto $])[$ Phyto $]-g_{\max } \frac{[\text { Phyto }]^{2}}{k_{\text {Phyto }}+[\text { Phyto }]^{2}}[$ Zoo $] ;$

$$
\frac{\partial[Z o o]}{\partial t}+\frac{\partial u[Z o o]}{\partial x}+\frac{\partial w[Z o o]}{\partial z}=\frac{\partial}{\partial x}\left(D_{x} \frac{\partial[Z o o]}{\partial x}\right)+\frac{\partial}{\partial z}\left(D_{z} \frac{\partial[Z o o]}{\partial z}\right)+
$$

$+g_{\max } \frac{[\text { Phyto }]^{2}}{k_{\text {Phyto }}+[\text { Phyto }]^{2}} \beta[$ Zoo $]-l_{B M}[$ Zoo $]-l_{E} \frac{[\text { Phyto }]^{2}}{k_{\text {Phyto }}+[\text { Phyto }]^{2}} \beta[$ Zoo $]-m_{\text {Zoo }}[\text { Zoo }]^{2}$;

$$
\begin{gathered}
\frac{\partial[S D N]}{\partial t}+\frac{\partial u[S D N]}{\partial x}+\frac{\partial w[S D N]}{\partial z}= \\
=\frac{\partial}{\partial x}\left(D_{x} \frac{\partial[S D N]}{\partial x}\right)+\frac{\partial}{\partial z}\left(D_{z} \frac{\partial[S D N]}{\partial z}\right)+g_{\max } \frac{[P h y t o]^{2}}{k_{\text {Phyto }}+[\text { Phyto }]^{2}}(1-\beta)[Z o o]+ \\
+m_{\text {Zoo }}[\text { Zoo }]^{2}+m_{\text {Phyto }}[\text { Phyto }]-\tau([S D N]+[\text { Phyto }])[S D N]-r_{S D N}[S D N]
\end{gathered}
$$




$$
\begin{gathered}
\frac{\partial[L D N]}{\partial t}+\frac{\partial u[L D N]}{\partial x}+\frac{\partial w[L D N]}{\partial z}= \\
=\frac{\partial}{\partial x}\left(D_{x} \frac{\partial[L D N]}{\partial x}\right)+\frac{\partial}{\partial z}\left(D_{z} \frac{\partial[L D N]}{\partial z}\right)+\tau([S D N]+[P h y t o])^{2}-r_{L D N}[L D N] \\
\frac{\partial[S D P]}{\partial t}+\frac{\partial u[S D P]}{\partial x}+\frac{\partial w[S D P]}{\partial z}= \\
=\frac{\partial}{\partial x}\left(D_{x} \frac{\partial[S D P]}{\partial x}\right)+\frac{\partial}{\partial z}\left(D_{z} \frac{\partial[S D P]}{\partial z}\right)+g_{\max } \frac{[P h y t o]^{2}}{k_{P h y t o}+[P h y t o]^{2}} r_{P N}(1-\beta)[Z o o]+ \\
+m_{Z o o} r_{P N}[Z o o]^{2}+m_{P h y t o} r_{P N}[P h y t o]-\tau([S D N]+[P h y t o])[S D P]-r_{S D P}[S D P] \\
\frac{\partial[L D P]}{\partial t}+\frac{\partial u[L D P]}{\partial x}+\frac{\partial w[L D P]}{\partial z}=\frac{\partial}{\partial x}\left(D_{x} \frac{\partial[L D P]}{\partial x}\right)+\frac{\partial}{\partial z}\left(D_{z} \frac{\partial[L D P]}{\partial z}\right)+ \\
+\tau([S D N]+[P h y t o])\left([S D P]+r_{P N}[P h y t o]\right)-r_{L D P}[L D P],
\end{gathered}
$$

где $[N],[A],[P],[C h l],[P h y t o],[Z o o]-$ концентрация нитратов $\left(\mathrm{NO}_{3}\right)$, аммония $\left(\mathrm{NH}_{4}\right)$, фосфатов $\left(\mathrm{PO}_{4}\right)$, хлорофилла $a$, фитопланктона, зоопланктона соответственно; $[S D N]$ и $[S D P]$ - концентрация мелкого детрита для $\mathrm{NO}_{3}$ и $\mathrm{PO}_{4}$ соответственно; $[L D N]$ и $[L D P]$ - концентрация крупного детрита для $\mathrm{NO}_{3}$ и $\mathrm{PO}_{4}$ соответственно; $u$ и $v$ - горизонтальная и вертикальная составляющие скорости соответственно; $D_{x}$ и $D_{z}-$ коэффициенты турбулентной диффузии в соответствующих направлениях.

Интенсивность роста фитопланктона определяется как

$$
\mu=\mu_{\max } f(I)\left(\sigma_{N}\left(\frac{[N]}{k_{N}+[N]} \cdot \frac{1}{1+[A] / k_{A}}+\frac{[A]}{k_{A}+[A]}\right)+\sigma_{P} \frac{[P]}{k_{P}+[P]}\right),
$$

где $\mu_{\max }$ - максимальная скорость роста фитопланктона [13]:

$$
\mu_{\max }(T)=\mu_{0} \cdot 1.066^{T} .
$$

Коэффициенты $\sigma_{N}$ и $\sigma_{P}$ отвечают за лимитирование продуктивности водоема по основным биогенным элементам:

если $\frac{[N]}{k_{N}+[N]} \cdot \frac{1}{1+[A] / k_{A}}+\frac{[A]}{k_{A}+[A]}>\frac{[P]}{k_{P}+[P]}$, то $\sigma_{N}=0$ и $\sigma_{P}=1$ (фосфорное лимитирование),

в противном случае $-\sigma_{N}=1$ и $\sigma_{P}=0$ (азотное лимитирование).

Функция $f(I)$ представляет связь фотосинтеза и света:

$$
\begin{gathered}
f(I)=\frac{\alpha I}{\sqrt{\mu_{\max }^{2}+\alpha^{2} I^{2}}} ; \\
I=I_{s} \cdot P A R \cdot \exp \left\{-d\left(k_{\text {water }}+k_{\text {chl }} \int_{d}^{L_{z}} \operatorname{Chl}(z) d z\right)\right\},
\end{gathered}
$$

где $I_{S}$ - приходящий на поверхность воды свет; $P A R$ - доля света для фотосинтеза $=0.43$ [9]. Параметру $I_{S}$ в модели соответствует коротковолновая радиация [9], которая вычисляется по формуле (8) из [14]. 
Участвующая в синтезе хлорофилла доля роста фитопланктона определяется как

$$
\rho_{C h l}=\frac{\theta_{\max } \mu[\text { Phyto }]}{\alpha I[C h l]} .
$$

Скорость нитрификации вычисляется [15]:

$$
n=n_{\max }\left(1-\max \left[0, \frac{I-I_{0}}{k_{I}+I-I_{0}}\right]\right),
$$

где $n_{\max }$ - максимальная скорость нитрификации. Параметры, входящие в расчетные формулы экосистемного блока модели, приведены в таблице.

\begin{tabular}{|c|c|c|}
\hline $\begin{array}{l}\text { Обозна- } \\
\text { чение }\end{array}$ & Наименование & Значение \\
\hline$\mu_{0}$ & Скорость роста фитопланктона при $0{ }^{\circ} \mathrm{C}$ & $0.59 \mathrm{cyT}^{-1}$ \\
\hline$k_{\text {water }}$ & Коэффициент ослабления света для воды & $0.04 \mathrm{M}^{-1}$ \\
\hline$k_{C h l}$ & Коэффициент ослабления света для хлорофилла & $0.025(\mathrm{M \Gamma} \mathrm{Chl})^{-1} \cdot \mathrm{M}^{-2}$ \\
\hline$\alpha$ & Начальная крутизна кривой $P-I$ & $\begin{array}{l}0.025 \text { мГ С }(\text { мГ Chl } \times \\
\quad \times \mathrm{BT}^{-2} \mathrm{M}^{-2} \cdot \mathrm{cyT}^{-1}\end{array}$ \\
\hline$\theta_{\max }$ & $\begin{array}{l}\text { Максимальное соотношение хлорофилла к биомассе } \\
\text { планктона }\end{array}$ & 0.054 мг Chl (мг C) $)^{-1}$ \\
\hline$r_{P N}$ & Клеточное соотношение P:N & 0.0625 \\
\hline$k_{N}$ & Константа полунасыщения фитопланктона по $\mathrm{NO}_{3}$ & 0.8 ммоль $\cdot \mathrm{m}^{-3}$ \\
\hline$k_{A}$ & Константа полунасыщения фитопланктона по $\mathrm{NH}_{4}$ & 0.8 ммоль $\cdot \mathrm{M}^{-3}$ \\
\hline$k_{P}$ & Константа полунасыщения фитопланктона по $\mathrm{PO}_{4}$ & 0.05 ммоль $\cdot \mathrm{M}^{-3}$ \\
\hline$m_{\text {Phyto }}$ & Смертность фитопланктона & 0.15 cyT $^{-1}$ \\
\hline$g_{\max }$ & Максимальная скорость питания зоопланктона & $0.6 \mathrm{cys}^{-1}$ \\
\hline$\beta$ & Эффективность усвоения зоопланктона по азоту & 0.75 \\
\hline$k_{\text {Phyto }}$ & Константа полунасыщения питания фитопланктона & 1 ммоль $\mathrm{N} \cdot \mathrm{M}^{-3}$ \\
\hline$l_{B M}$ & $\begin{array}{l}\text { Скорость экскреции зоопланктона за счет основного об- } \\
\text { мена }\end{array}$ & $0.1 \mathrm{cyT}^{-1}$ \\
\hline$l_{E}$ & $\begin{array}{l}\text { Скорость экскреции зоопланктона за счет видовой осо- } \\
\text { бенности }\end{array}$ & $0.1 \mathrm{cyT}^{-1}$ \\
\hline$m_{Z o o}$ & Смертность зоопланктона & $\begin{aligned} & 0.025 \mathrm{cyт}^{-1} \times \\
& \times\left(\text { ммоль } \mathrm{N} \cdot \mathrm{M}^{-3}\right)^{-1} \\
&\end{aligned}$ \\
\hline$r_{S D N}$ & Скорость реминерализации мелкого детрита для азота & $0.03 \mathrm{cyT}^{-1}$ \\
\hline$r_{L D N}$ & Скорость реминерализации крупного детрита для азота & $0.01 \mathrm{cyT}^{-1}$ \\
\hline$r_{S D P}$ & Скорость реминерализации мелкого детрита для фосфора & $0.075 \mathrm{cyT}^{-1}$ \\
\hline$r_{L D P}$ & Скорость реминерализации крупного детрита для фосфора & $0.025 \mathrm{cyT}^{-1}$ \\
\hline$\tau$ & Скорость коагуляции & $0.05 \mathrm{cys}^{-1}$ \\
\hline$n_{\max }$ & Максимальная скорость нитрификации & $0.05 \mathrm{cyT}^{-1}$ \\
\hline$I_{0}$ & Порог ФАР для ингибирования процесса нитрификации & $0.0095 \mathrm{BT} \cdot \mathrm{M}^{-2}$ \\
\hline$k_{I}$ & $\begin{array}{l}\text { Полунасыщение ФАР для ингибирования процесса нит- } \\
\text { рификации }\end{array}$ & $0.1 \mathrm{BT} \cdot \mathrm{M}^{-2}$ \\
\hline
\end{tabular}

Значения биогеохимических параметров модели

Для биологических переменных модели заданы следующие начальные условия:

- значения концентрации нитратов, аммония, фосфатов, зоопланктона и хлорофилла $а$ приняты равными $5.0,4.0,0.4,0.3$ и 0.3 ммоль $\mathrm{N} \mathrm{M}^{-3}$ соответственно; 
- концентрация для всех детритных компонентов в начальный момент времени составляет 0.1 ммоль $\mathrm{N} \cdot \mathrm{M}^{-3}$;

- значение биомассы фитопланктона задано исходя из соотношения 1.59 для $[$ Chl $] /[$ Phyto $]$.

Граничные условия для уравнений модели имеют вид:

а) на поверхности водоема

$$
\begin{gathered}
\frac{\partial[N]}{\partial z}=0 ; \frac{\partial[A]}{\partial z}=0 ; \frac{\partial[P]}{\partial z}=0 ; \frac{\partial[C h l]}{\partial z}=0 ; \frac{\partial[\text { Phyto }]}{\partial z}=0 ; \\
\frac{\partial[Z o o]}{\partial z}=0 ; \frac{\partial[S D N]}{\partial z}=0 ; \frac{\partial[L D N]}{\partial z}=0 ; \frac{\partial[S D P]}{\partial z}=0 ; \frac{\partial[L D P]}{\partial z}=0 ;
\end{gathered}
$$

б) на твёрдых границах

$$
\begin{gathered}
\frac{\partial[N]}{\partial n}=0 ; \frac{\partial[A]}{\partial n}=0 ; \frac{\partial[P]}{\partial n}=0 ; \frac{\partial[C h l]}{\partial n}=0 ; \frac{\partial[\text { Phyto }]}{\partial n}=0 ; \\
\frac{\partial[Z o o]}{\partial n}=0 ; \frac{\partial[S D N]}{\partial n}=0 ; \frac{\partial[L D N]}{\partial n}=0 ; \frac{\partial[S D P]}{\partial n}=0 ; \frac{\partial[L D P]}{\partial n}=0,
\end{gathered}
$$

где $n$ - направление внешней нормали к области;

в) на границе впадения реки в озеро

$[N]=5.0$ ммоль $\mathrm{N} \cdot \mathrm{M}^{-3} ;[A]=4.0$ ммоль $\mathrm{N} \cdot \mathrm{M}^{-3} ;[P]=0.4$ ммоль $\mathrm{N} \cdot \mathrm{м}^{-3} ;[Z o o]=$ $=0.3$ ммоль $\mathrm{N} \cdot \mathrm{M}^{-3}$;

$[C h l]=0.3$ ммоль $\mathrm{N} \cdot \mathrm{M}^{-3} ;[S D N]=0.1$ ммоль $\mathrm{N} \cdot \mathrm{M}^{-3} ;[L D N]=0.1$ ммоль $\mathrm{N} \cdot \mathrm{M}^{-3}$; $[S D P]=0.1$ ммоль $\mathrm{N} \cdot \mathrm{M}^{-3}$;

$[L D P]=0.1$ ммоль $\mathrm{N} \cdot \mathrm{M}^{-3}$;

г) на открытой (правой) границе задаются условия радиационного типа:

$$
\begin{gathered}
\frac{\partial \phi}{\partial t}+c_{\phi} \frac{\partial \phi}{\partial x}=0 \\
(\phi=[N],[A],[P],[C h l],[\text { Phyto }],[Z o o],[S D N],[L D N],[S D P],[L D P]),
\end{gathered}
$$

фазовая скорость $c_{\phi}$ рассчитывается из пространственных и временных тенденций внутри области [16].

Условную запись разностной схемы для конечного объёма $P$ для уравнений (1) - (10) можно представить в обобщенном виде:

$$
\begin{gathered}
\frac{\Phi_{P}-\Phi_{P}^{0}}{\Delta t}=\frac{1}{2}\left[-\left(C_{x}^{*}\right)_{P}-\left(C_{z}^{*}\right)_{P}+\left(D_{x}^{*}\right)_{P}+\left(D_{z}^{*}\right)_{P}+\left(S_{\Phi}\right)_{P}\right]^{0}+ \\
+\frac{1}{2}\left[-\left(C_{x}^{*}\right)_{P}-\left(C_{z}^{*}\right)_{P}+\left(D_{x}^{*}\right)_{P}+\left(D_{z}^{*}\right)_{P}+\left(S_{\Phi}\right)_{P}\right],
\end{gathered}
$$

где $\Phi=[N],[A],[P],[C h l],[P h y t o],[Z o o],[S D N],[L D N],[S D P],[L D P] ; \quad\left(C_{x}^{*}\right)_{P}$, $\left(C_{z}^{*}\right)_{P}$ и $\left(D_{x}^{*}\right)_{P},\left(D_{z}^{*}\right)_{P}$ - аппроксимация конвективных и диффузионных членов соответственно; $\left(S_{\Phi}\right)_{P}$ - источниковый член; $\Phi_{P}^{0}$ соответствует значению $\Phi$ в центре конечного объёма на временном слое $t$, а $\Phi_{P}-$ на слое $t+\Delta t$. Видно, что нестационарный член в (17) аппроксимируется схемой Кранка - Николсон. Для аппроксимации диффузионных членов используется центрально-разностная схема, а конвективных членов - противопотоковая схема QUICK второго порядка точности [17]. 
Конечная система алгебраических уравнений, соответствующая дифференциальной задаче имеет вид

$$
a_{P} \Phi_{P}=a_{E} \Phi_{E}+a_{W} \Phi_{W}+a_{N} \Phi_{N}+a_{S} \Phi_{S}+b,
$$

где индекс $P$ обозначает центр текущего конечного объёма $(i, j)$, а индексы $E, W, N$ и $S$ (согласно «географической» системе обозначений) - центры соседних объёмов $(i+1, j),(i-1, j),(i, j+1)$ и $(i, j-1)$ соответственно; $a_{E} \geq 0, a_{W} \geq 0, a_{N} \geq 0, a_{S} \geq 0$ и $a_{P}=a_{E}+a_{W}+a_{N}+a_{S}+a_{P}^{0}>0\left(a_{P}^{0} \equiv \frac{\Delta x \Delta z}{\Delta t}\right)$. Решение системы (18) осуществляется методом неполной факторизации, устойчивость которого обеспечивается диагональным преобладанием [18].

Важно заметить, что линеаризация источникового члена $\left(S_{\Phi}\right)_{P}$ осуществляется следующим образом [19]:

$$
\left(S_{\Phi}\right)_{P} \approx S_{C}+S_{P} \Phi_{P}
$$

где $S_{C}$ - постоянная составляющая $\left(S_{\Phi}\right)_{P}$, а $S_{P}$ - коэффициент ( $S_{P}$ не есть значение $\left(S_{\Phi}\right)_{P}$ в точке $\left.P\right)$. Если коэффициент $S_{P}$ всегда отрицателен или меняет знак, то для усиления диагонального преобладания матрицы коэффициентов системы (18) переносится в левую часть уравнения (18). Детальное описание численного метода решения уравнений вида (18) приведено в работах $[6,20]$.

Коэффициенты вертикальной диффузии определяются как

$$
D_{z}=\frac{v_{T}}{\operatorname{Pr}_{T}}+\frac{v}{\operatorname{Pr}},
$$

где $v_{T}$ - турбулентная кинематическая вязкость (рассчитывается по $k$ - $\omega$-модели Уилкокса [22]); $v$ - молекулярная кинематическая вязкость воды; $\operatorname{Pr}$ и $\operatorname{Pr}_{T}-$ молекулярное и турбулентное числа Прандтля.

Коэффициенты горизонтальной диффузии задаются постоянными [22]:

$$
D_{x}=2.5 \mathrm{~m}^{2} / \mathrm{c} .
$$

Расчеты проводились на серверной платформе Supermicro с графическим ускорителем Tesla C2050.

\section{Область исследования}

Областью исследования является вертикальное сечение Баргузинского залива озера Байкал, начало системы координат совпадает с устьем р. Баргузин 5325'30" с.ш., 108 59'50" в. д. [23] (рис. 2, a). Данные по рельефу дна взяты из работы [24] (рис. 2,b).

Вычислительная область имеет протяженность 20 км и глубину 100 м (рис. 2, b). Глубина открытого участка речного стока (на левой границе) составляет 7.5 м. Расчётная область покрыта равномерной ортогональной сеткой с шагами $h_{x}=25$ м и $h_{z}=2.5$ м. Шаг по времени - 30 с. Исследование сходимости численного решения уравнений вида (18) по шагам сетки и времени проводилось в работах $[20,25,26]$. В качестве критерия сходимости итерационного процесса использовалось условие малости невязки $\left(<\varepsilon=10^{-10}\right)$. Вычисления проводились для одного варианта расчетной сетки. 


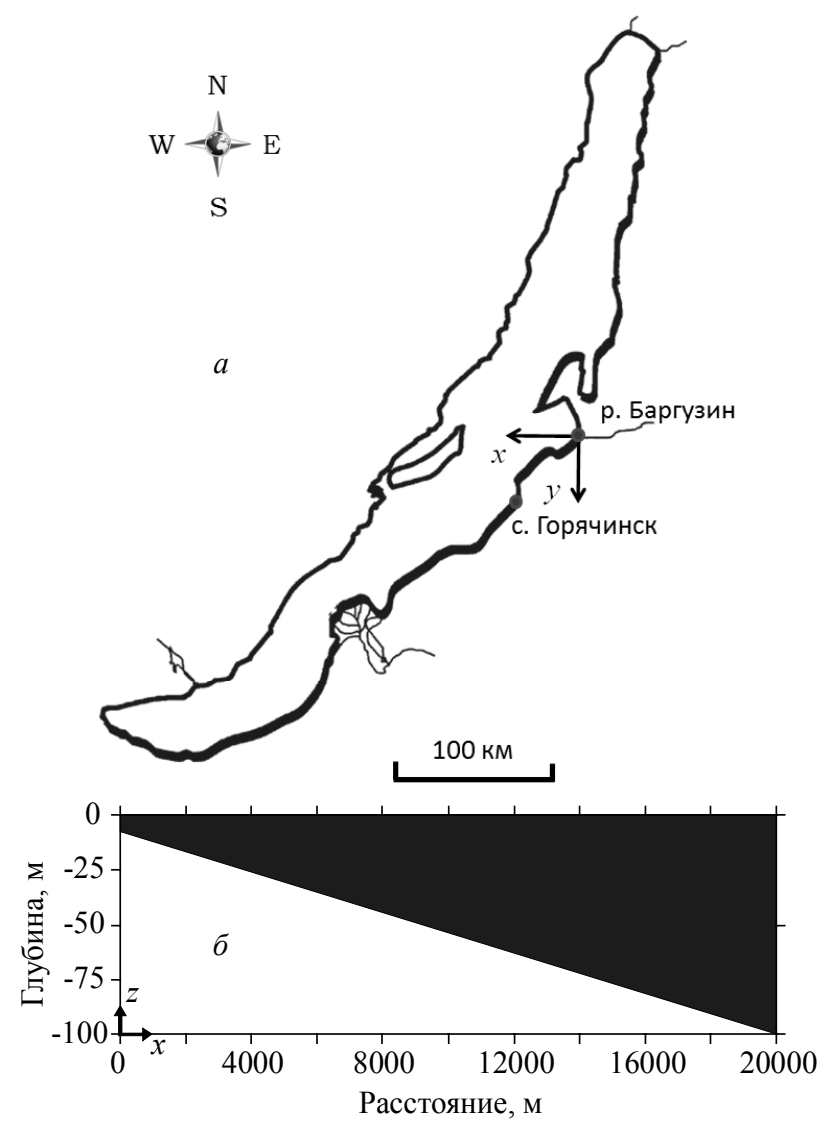

Рис. 2. Озеро Байкал: $a$ - схема разреза, $b$ - геометрия расчетной области

Fig. 2. Lake Baikal: $a$-cross-section scheme, $b$-calculation domain

Начальное значение температуры воды в озере задано равным $4.5{ }^{\circ} \mathrm{C}$. Температура воды в устье р. Баргузин монотонно растет с 12 до $15^{\circ} \mathrm{C}$. Минерализация воды в озере составляет 96 мг/кг [27], в реке - 128.2 мг/л (соответствует среднему значению по всем притокам озера Байкал [28]). Скорость течения р. Баргузин при впадении в озеро $-0.5 \mathrm{~cm} / \mathrm{c}$. Компоненты тепловых потоков, поступающих на водное зеркало, вычислены по расчетным формулам, приведенным в работе [29], на основе данных о температуре воздуха, относительной влажности, атмосферному давлению и облачности из архива погодных условий метеостанции с. Горячинск в период с 1 по 31 августа 2018 г.

\section{Результаты моделирования и их обсуждение}

Распределение хлорофилла $a$ (основного пигмента, жизненно необходимого для фотосинтеза в растительных клетках), полученное в результате численного моделирования (рис. $3, a$ ) на рассматриваемом разрезе оз. Байкал, имеет сложную структуру. В приустьевой области водоема наблюдается высокая концентрация хлорофилла (изолинии вертикально-наклонные), максимальное значение приходится на границе раздела река - озеро. Данные полевых измерений $[4,30]$ также 
указывают на повышенное содержание первичной продукции в районе впадения р. Баргузин в летний период. В открытой части водоема концентрация хлорофилла имеет горизонтально однородное распределение с локальным максимумом ( $>0.06$ ммоль $\mathrm{M}^{-3}$ ) на глубине 10-30 м, причем с увеличением времени моделирования её изменение в вертикальном направлении усиливается (рис. 3, a2). Эти результаты качественно согласуются с распределением хлорофилла $a$, полученным методом спектрофотометрии в центральной части Баргузинского залива 24-31 августа 2002 г.: пиковое значение хлорофилла детектировано на глубине 10 м [4].
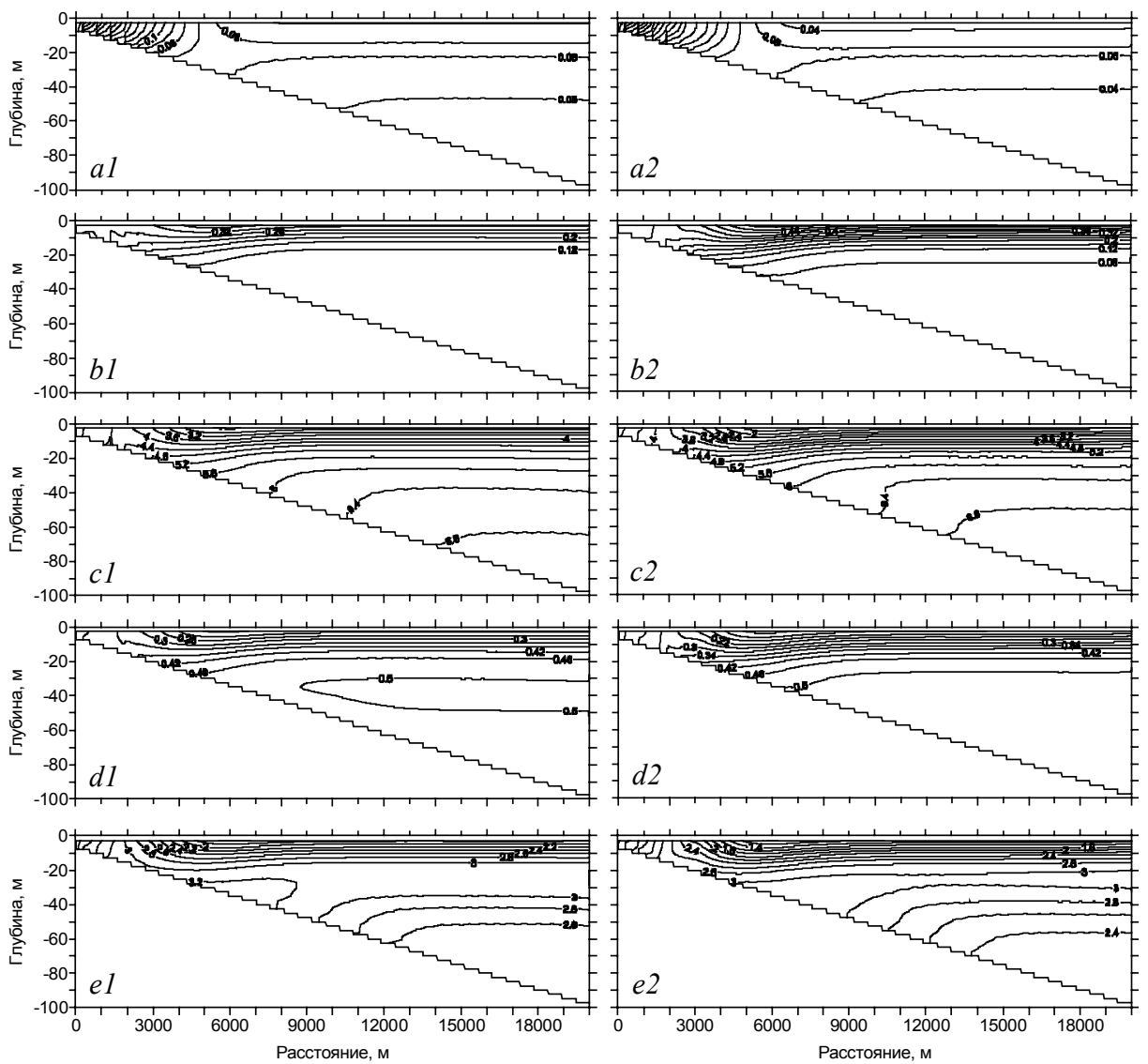

Рис. 3. Распределение хлорофилла $a\left[\mathrm{мг} \cdot \mathrm{M}^{-3}\right](a)$, зоопланктона [ммоль $\left.\mathrm{N} \cdot \mathrm{M}^{-3}\right](b)$, нитрата $\left[\right.$ ммоль $\left.\cdot \mathrm{m}^{-3}\right](c)$, фосфата [ммоль $\left.\cdot \mathrm{M}^{-3}\right](d)$ и аммония [ммоль $\left.\cdot \mathrm{M}^{-3}\right](e)$ на 24-е $(1)$ и 28-е (2) сутки моделирования

Fig. 3. Distribution of chlorophyll $a\left[\mathrm{mg} \cdot \mathrm{m}^{-3}\right](a)$, zooplankton $\left[\mathrm{mmol} \mathrm{N} \cdot \mathrm{m}^{-3}\right](b)$, nitrate $\left[\mathrm{mmol} \cdot \mathrm{m}^{-3}\right](c)$, phosphate $\left[\mathrm{mmol} \cdot \mathrm{m}^{-3}\right](d)$, ammonium $\left[\mathrm{mmol} \cdot \mathrm{m}^{-3}\right](e)$ on the $24(1)$ and 28 (2) days

Зоопланктон сосредоточен в верхней 25-метровой толще водоема (рис. $3, b$ ), его наибольшая концентрация отмечается в приповерхностном слое. В открытой части водоема (по сравнению с приустьевым пространством) рост биомассы зоопланктона происходит интенсивнее (рис. $3, b$ ). Такая картина развития зоопланктонных популяций в конце лета имеет качественное и количественное различие от 
весенней динамики, связанной с существованием термобара [31, 32]. На 28-й день концентрация зоопланктона возрастает до 0.52 ммоль $\mathrm{N} \cdot \mathrm{M}^{-3}$ (рис. $3, b 2$ ).

Содержание биогенных элементов в воде имеет обратную корреляцию с распределением биомассы планктона: на участках водоема с высокой концентрацией фито- и зоопланктона наблюдается снижение $\mathrm{NO}_{3}, \mathrm{PO}_{4}$ и $\mathrm{NH}_{4}$ (рис. $3, c, d, e$ ). Результаты моделирования показывают, что уменьшение количества $\mathrm{NO}_{3}$ в присклоновой области водоема происходит быстрее, чем в пелагической зоне (рис. 3, c), при этом развитие гидробиологических процессов ведет к усилению данной тенденции (рис. $3, c 2)$. Повышенное содержание $\mathrm{PO}_{4}\left(>0.5\right.$ ммоль $\left.\cdot \mathrm{M}^{-3}\right)$ на 24-й день заметно в 30-50-метровом слое (рис. 3, d1). На 28 день $\mathrm{PO}_{4}$ (рис. 3, d2) в водах открытого озера принимает горизонтально однородное распределение, количество $\mathrm{PO}_{4}$ возрастает с глубиной. Наибольшая концентрация $\mathrm{NH}_{4}$ отмечается в 20-30-метровой толще (рис. 3,e), выше и ниже указанного слоя происходит уменьшение количества $\mathrm{NH}_{4}$. На 24-й день наблюдается образование «языка» с высоким содержанием $\mathrm{NH}_{4}$ у нижней границы рассматриваемой области на расстоянии 4.5-8.5 км от устья речного притока (рис. $3, e 1$ ). Мониторинговые исследования химического состава воды в оз. Байкал в летний период также фиксируют:

1) различие в содержании биогенных элементов в мелководных и пелагических участках озера (2002-2007 гг.) [33];

2) увеличение концентрации нитратов и фосфатов с ростом глубины в пелагиали (1993-2011 гг.) [34].

\section{Заключение}

Построенная математическая модель позволяет воспроизводить биогеохимические процессы в пресноводном озере. Проведенные на примере Баргузинского залива оз. Байкал расчеты показали, что в последней декаде августа

1) повышенное содержание хлорофилла $a$ в пелагиали водоема локализовано на глубине 10-30 м;

2) зоопланктон сосредоточен в верхнем 25-метровом слое, причем в открытой части озера увеличение его биомассы происходит быстрее;

3) на участках водоема с высокой концентрацией фито- и зоопланктона наблюдается снижение количества биогенных элементов.

Полученные результаты качественно согласуются с данными мониторинговых исследований химического состава воды в озере Байкал в летний период.

\section{ЛИТЕРАТУРА}

1. Weiss R.F., Carmack E.C., Koropalov V.M. Deep-water renewal and biological production in Lake Baikal // Nature. 1991. V. 349. P. 665-669. DOI: 10.1038/349665a0.

2. Watanabe Y., Drucker V.V. Phytoplankton blooms in Lake Baikal, with reference to the lake's present state of eutrophication // Ancient lakes: their cultural and biological diversity / Kawanabe H., Goldman G.W., Roosevelt A.C. (eds). Kenobi Productions, Belgium. 1999. P. 217-225.

3. Goldman C.R., Elser J.J., Richards R.C., Reuter J.E., Priscu J.C., Levin A.L. Thermal stratification, nutrient dynamics, and phytoplankton productivity during the onset of spring phytoplankton growth in Lake Baikal, Russia // Hydrobiologia. 1996. V. 331. No. 1-3. P. 9-24. DOI: $10.1007 / \mathrm{BF} 00025403$.

4. Satoh Y., Katano T., Satoh T., Mitamura O., Anbutsu K., Nakano S.-I., Ueno H., Kihira M., Drucker V., Tanaka Y., Mimura, T. Watanabe Y., Sugiyama M. Nutrient limitation of the primary production of phytoplankton in Lake Baikal // Limnology. 2006. V. 7. No. 3. P. 225229. DOI: $10.1007 / \mathrm{s} 10201-006-0187-8$. 
5. Henderson-Sellers B. Engineering Limnology. London: Pitman, 1984. 356 p.

6. Tsydenov B.O., Kay A., Starchenko A.V. Numerical modeling of the spring thermal bar and pollutant transport in a large lake // Ocean Modelling. 2016. V. 104. P. 73-83. DOI: 10.1016/j.ocemod.2016.05.009.

7. Tsydenov B.O. A numerical study of the thermal bar in shallow water during the autumn cooling // J. Great Lakes Res. 2019. V. 45. No. 3. P. 715-725. DOI: 10.1016/j.jglr. 2019.05.012.

8. Fasham M.J.R., Ducklow H.W., McKelvie S.M. A nitrogen-based model of plankton dynamics in the oceanic mixed layer // J. Mar. Res. 1990. V. 48. No. 3. P. 591-639. DOI: 10.1357/ 002224090784984678.

9. Fennel K., Wilkin J., Levin J., Moisan J., O'Reilly J., Haidvogel D. Nitrogen cycling in the Middle Atlantic Bight: Results from a three-dimensional model and implications for the North Atlantic nitrogen budget // Global Biogeochemical Cycles. 2006. V. 20. No. 3. GB3007. DOI: 10.1029/2005GB002456.

10. Hofmann E., Druon J.-N., Fennel K., Friedrichs M., Haidvogel D., Lee C., Mannino A., McClain C., Najjar R., O'Reilly J., Pollard D., Previdi M., Seitzinger S., Siewert J., Signorini S., Wilkin J. Eastern US continental shelf carbon budget: Integrating models, data assimilation, and analysis // Oceanography. 2008. V.21. No. 1. P. 86-104. DOI: 10.5670/ oceanog.2008.70.

11. Gan J., Lu Z., Cheung A., Dai M., Liang L., Harrison P.J., Zhao X. Assessing ecosystem response to phosphorus and nitrogen limitation in the Pearl River plume using the Regional Ocean Modeling System (ROMS) // J. Geophys. Res. C: Oceans. 2014. V. 119. No. 12. P. 8858-8877. DOI: 10.1002/2014JC009951.

12. Redfield A.C. The biological control of chemical factors in the environment // Am. Sci. 1958. No. 46. P. 205-211.

13. Eppley R.W. Temperature and phytoplankton growth in the sea // Fish. Bull. 1972. V. 70. No. 4. P. $1063-1085$.

14. Tsydenov B.O., Starchenko A.V. To the selection of heat flux parameterization models at the water-air interface for the study of the spring thermal bar in a deep lake // Proc. SPIE 9680, 96800H. 2015. P. 1-8. DOI: 10.1117/12.2205687.

15. Olson R. J. Differential photoinhibition of marine nitrifying bacteria: A possible mechanism for the formation of the primary nitrite maximum // J. Mar. Res. 1981. V. 39. No. 2. P. 227-238.

16. Orlanski I. A simple boundary condition for unbounded hyperbolic flows // J. Comput. Phys. 1976. V. 21. No. 3. P. 251-269. DOI: 10.1016/0021-9991(76)90023-1.

17. Leonard B. A stable and accurate convective modeling procedure based on quadratic upstream interpolation // Computer Methods in Applied Mechanics and Engineering. 1979. V. 19. No. 1. P. 59-98. DOI: 10.1016/0045-7825(79)90034-3.

18. Ильин В.П. Методы неполной факторизации для решения алгебраических систем. М.: Физматлит, 1995. 288 с.

19. Patankar $S$. Numerical heat transfer and fluid flow. CRC Press, 1980. 214 p.

20. Цыљенов Б.О. Численное моделирование эффекта весеннего термобара в глубоком озеpe: дис. ... канд. физ.-мат. наук. Томск, 2013. 145 с.

21. Wilcox D.C. Reassessment of the scale-determining equation for advanced turbulence models // AIAA J. 1988. V. 26. No. 11. P. 1299-1310. DOI: 10.2514/3.10041.

22. Holland P.R., Kay A., Botte V. Numerical modelling of the thermal bar and its ecological consequences in a river-dominated lake // J. Mar. Syst. 2003. V. 43. No. 1-2. P. 61-81. DOI: 10.1016/S0924-7963(03)00089-7.

23. Баргузин (река). URL: https://ru.wikipedia.org/wiki/\%D0\%91\%D0\%B0\%D1\%80\%D0\% $\mathrm{B} 3 \% \mathrm{D} 1 \% 83 \% \mathrm{D} 0 \% \mathrm{~B} 7 \% \mathrm{D} 0 \% \mathrm{~B} 8 \% \mathrm{D} 0 \% \mathrm{BD} \_(\% \mathrm{D} 1 \% 80 \% \mathrm{D} 0 \% \mathrm{~B} 5 \% \mathrm{D} 0 \% \mathrm{BA} \% \mathrm{D} 0 \% \mathrm{~B} 0)$ (дата обращения: 19.01.2020).

24. Ueno H., Katano T., Nakano S.-I., Mitamura O., Anbutsu K., Satoh Y., Drucker V., Sugiyama $M$. Abundance and community structure of picoplankton and protists in the microbial food web of Barguzin Bay, Lake Baikal. Aquatic Ecology. 2005. V. 39. No. 3. P. 263-270. DOI: 10.1007/s10452-005-6057-3. 
25. Цыљенов Б.О. Численное исследование распространения примеси в пресном озере на основе распределения мутности воды // Вычислительные технологии. 2017. Т. 22. № 1. C. $113-124$.

26. Tsydenov B.O. Simulating phytoplankton growth during the spring thermal bar in a deep lake // J. Mar. Syst. 2019. V. 195. P. 38-49. DOI: 10.1016/j.jmarsys.2019.03.009.

27. Shimaraev M.N., Verbolov V.I., Granin N.G., Sherstyankin P.P. Physical Limnology of Lake Baikal: A Review. Irkutsk-Okayama, 1994. 81 p.

28. Вотинщев К.К. Гидрохимия // Проблемы Байкала / отв. ред. Г.И. Галазий, К.К. Вотинцев. Новосибирск, 1978. Т. 16(36). С. 124-146.

29. Tsydenov B.O. Numerical modeling of the effect of inflow water mineralization in the dynamics of the autumnal thermal bar in Kamloops Lake // Moscow Univ. Phys. Bull. V. 73. No. 4. P. 435-440. DOI: 10.3103/S0027134918040148.

30. Katano T., Nakano S.-I., Ueno H., Mitamura O., Anbutsu K., Kihira M., Satoh Y., Satoh T., Drucker V.V., Tanaka Y., Akagashi Y., Sugiyama M. Abundance and composition of the summer phytoplankton community along a transect from the Barguzin River to the central basin of Lake Baikal // Limnology. 2008. V. 9. No. 3. P. 243-250. DOI: 10.1007/s10201-0080252-6.

31. Tsydenov B.O. Numerical modeling of the autumnal thermal bar// J. Mar. Syst. 2018. V. 179. P. 1-9. DOI: 10.1016/j.jmarsys.2017.11.004.

32. Цыденов Б.О. Численное моделирование весенней динамики планктона на примере Селенгинского мелководья оз. Байкал // Труды КарНЦ РАН. 2019. № 3. С. 114-123. DOI: 10.17076/lim948.

33. Tomberg I.V., Sorokovikova L.M., Popovskaya G.I., Bashenkhaeva N.V., Sinyukovich V.N., Ivanov V.G. Concentration dynamics of biogenic elements and phytoplankton at Selenga R. Mouth and in Selenga shallows (Lake Baikal) // Water Resources. 2014. V. 41. No. 6. P. 687695. DOI: 10.1134/S0097807814050157.

34. Домымева В.М., Шимараев М.Н., Сакирко М.В., Онищук Н.А. Динамика концентрации биогенных элементов и растворенных газов в воде озера Байкал в современный период // Органическое вещество и биогенные элементы во внутренних водоемах и морских водах: Материалы V Всерос. симп. с междунар. участием (Петрозаводск, 10-14 сент. 2012 г.). Петрозаводск, 2012. С. 23-26.

Статья поступила 21.01.2020 г.

Tsydenov B.O. (2020) A MATHEMATICAL MODEL FOR SIMULATING THE BIOGEOCHEMICAL PROCESSES IN A FRESHWATER LAKE. Vestnik Tomskogo gosudarstvennogo universiteta. Matematika i mekhanika [Tomsk State University Journal of Mathematics and Mechanics]. 65. pp. 53-67

DOI $10.17223 / 19988621 / 65 / 4$

Keywords: mathematical model, freshwater lake ecosystem, numerical experiment, Lake Baikal.

The most significant chemical elements in a lake ecosystem are phosphorus and nitrogen because one of them is the limiting factor of primary production rate. The phosphorus content level in a freshwater lake is of substantive importance in prediction of phytoplankton blooms. In that regard, the problem of the development of integrative models aimed at numerical simulation of the biochemical processes of phosphorus and nitrogen limitation in am aquatic ecosystem is topical for the contemporary stage of the development of mathematical methods in problems of limnology.

In this paper, a mathematical model for studying the environmental status of a freshwater lake is proposed. The model includes ten prognostic variables: nitrate, phosphate, ammonium, chlorophyll $a$, phytoplankton, zooplankton, small nitrate detritus, large nitrate detritus, small phosphate detritus, and large phosphate detritus. Calculations performed based on the model developed for Barguzin Bay of Lake Baikal showed that during last ten days of August: 
- increased chlorophyll $a$ content in the pelagic zone of the lake is localized at depths of 10 $30 \mathrm{~m}$;

- zooplankton is concentrated in the upper $25 \mathrm{~m}$ layer, and its biomass grows faster in the open water area;

- the decrease in nutrients is registered in the zones with high phyto- and zooplankton populations.

The results obtained are in qualitative agreement with data from monitoring studies of the chemical composition of water in Lake Baikal in summer.

Financial support. The reported study was funded by RFBR, project number 19-31-60003.

AMS Mathematical Subject Classification: 93A30

Bair O. TSYDENOV (Candidate of Physics and Mathematics, V.E. Zuev Institute of Atmospheric Optics of Siberian Branch of the Russian Academy of Science, Tomsk, Russia; Tomsk State University, Tomsk, Russia). E-mail: btsydenov@gmail.com

\section{REFERENCES}

1. Weiss R.F., Carmack E.C., Koropalov V.M. (1991) Deep-water renewal and biological production in Lake Baikal. Nature. 349. pp. 665-669. DOI: 10.1038/349665a0.

2. Watanabe Y., Drucker V.V. (1999) Phytoplankton blooms in Lake Baikal, with reference to the lake's present state of eutrophication. Ancient lakes: their cultural and biological diversity (Kawanabe H., Goldman G.W., Roosevelt A.C., eds.). Kenobi Productions, Belgium. pp. $217-225$.

3. Goldman C.R., Elser J.J., Richards R.C., Reuter J.E., Priscu J.C., Levin A.L. (1996) Thermal stratification, nutrient dynamics, and phytoplankton productivity during the onset of spring phytoplankton growth in Lake Baikal, Russia. Hydrobiologia. 331 (1-3). pp. 9-24. DOI: 10.1007/BF00025403.

4. Satoh Y., Katano T., Satoh T., Mitamura O., Anbutsu K., Nakano S.-I., Ueno H., Kihira M., Drucker V., Tanaka Y., Mimura, T. Watanabe Y., Sugiyama M. (2006) Nutrient limitation of the primary production of phytoplankton in Lake Baikal. Limnology 7(3). pp. 225-229. DOI: 10.1007/s10201-006-0187-8.

5. Henderson-Sellers B. (1984) Engineering Limnology. London: Pitman.

6. Tsydenov B.O., Kay A., Starchenko A.V. (2016) Numerical modeling of the spring thermal bar and pollutant transport in a large lake. Ocean Modelling. 104. pp. 73-83. DOI: 10.1016/j.ocemod.2016.05.009.

7. Tsydenov B.O. (2019) A numerical study of the thermal bar in shallow water during the autumn cooling. J. Great Lakes Res. 45(4). pp. 715-725. DOI: 10.1016/j.jglr.2019.05.012.

8. Fasham M.J.R., Ducklow H.W., McKelvie S.M. (1990) A nitrogen-based model of plankton dynamics in the oceanic mixed layer. J. Mar. Res. 48(3). pp. 591-639. DOI: 10.1357/ 002224090784984678.

9. Fennel K., Wilkin J., Levin J., Moisan J., O’Reilly J., Haidvogel D. (2006) Nitrogen cycling in the Middle Atlantic Bight: Results from a three-dimensional model and implications for the North Atlantic nitrogen budget. Global Biogeochemical Cycles. 20(3). GB3007. DOI: 10.1029/2005GB002456.

10. Hofmann E., Druon J.-N., Fennel K., Friedrichs M., Haidvogel D., Lee C., Mannino A., McClain C., Najjar R., O’Reilly J., Pollard D., Previdi M., Seitzinger S., Siewert J., Signorini S., Wilkin J. (2008) Eastern US continental shelf carbon budget: Integrating models, data assimilation, and analysis. Oceanography 1. pp. 86-104. DOI: 10.5670/oceanog.2008.70.

11. Gan J., Lu Z., Cheung A., Dai M., Liang L., Harrison P.J., Zhao X. (2014) Assessing ecosystem response to phosphorus and nitrogen limitation in the Pearl River plume using the Regional Ocean Modeling System (ROMS) // J. Geophys. Res. C: Oceans 119(12). pp. 88588877. DOI: $10.1002 / 2014 J C 009951$.

12. Redfield A.C. (1958) The biological control of chemical factors in the environment. Am. Sci. 46. pp. 205-211. 
13. Eppley R.W. (1972) Temperature and phytoplankton growth in the sea. Fish. Bull. 70(4). pp. 1063-1085.

14. Tsydenov B.O., Starchenko A.V. (2015) To the selection of heat flux parameterization models at the water-air interface for the study of the spring thermal bar in a deep lake. Proc. SPIE 9680, 96800H. pp. 1-8. DOI: 10.1117/12.2205687.

15. Olson R.J. (1981) Differential photoinhibition of marine nitrifying bacteria: A possible mechanism for the formation of the primary nitrite maximum. J. Mar. Res. 39(2). pp. 227238.

16. Orlanski I. (1976) A simple boundary condition for unbounded hyperbolic flows. J. Comput. Phys. 21(3). pp. 251-269. DOI: 10.1016/0021-9991(76)90023-1.

17. Leonard B. (1979) A stable and accurate convective modeling procedure based on quadratic upstream interpolation. Computer Methods in Applied Mechanics and Engineering. 19(1). pp. 59-98. DOI: 10.1016/0045-7825(79)90034-3.

18. Il'in V.P. (1995) Metody nepolnoy faktorizatsii dlya resheniya algebraicheskikh sistem [Incomplete factorization methods for solving algebraic systems]. Moscow: Fizmatlit.

19. Patankar S. (1980) Numerical heat transfer and fluid flow. Boca Raton: CRC Press.

20. Tsydenov B.O. (2013) Chislennoe modelirovanie effekta vesennego termobara $v$ glubokom ozere [Numerical modeling of the effect of the spring thermal bar in a deep lake]. Physics and Mathematics Cand. Diss. Tomsk.

21. Wilcox D.C. (1988) Reassessment of the scale-determining equation for advanced turbulence models. AIAA J. 26(11). pp. 1299-1310. DOI: 10.2514/3.10041.

22. Holland P.R., Kay A., Botte V. (2003) Numerical modelling of the thermal bar and its ecological consequences in a river-dominated lake. J. Mar. Syst. 43(1-2). pp. 61-81. DOI: 10.1016/S0924-7963(03)00089-7.

23. The Barguzin (river). URL: https://ru.wikipedia.org/wiki/\%D0\%91\%D0\%B0\%D1\%80\%D0 $\% \mathrm{~B} 3 \% \mathrm{D} 1 \% 83 \% \mathrm{D} 0 \% \mathrm{~B} 7 \% \mathrm{D} 0 \% \mathrm{~B} 8 \% \mathrm{D} 0 \% \mathrm{BD}(\% \mathrm{D} 1 \% 80 \% \mathrm{D} 0 \% \mathrm{~B} 5 \% \mathrm{D} 0 \% \mathrm{BA} \% \mathrm{D} 0 \% \mathrm{~B} 0)$.

24. Ueno H., Katano T., Nakano S.-I., Mitamura O., Anbutsu K., Satoh Y., Drucker V., Sugiyama M. (2005) Abundance and community structure of picoplankton and protists in the microbial food web of Barguzin Bay, Lake Baikal. Aquatic Ecology 39(3). pp. 263-270. DOI: 10.1007/s10452-005-6057-3.

25. Tsydenov B.O. (2017) Chislennoe issledovanie rasprostraneniya primesi v presnom ozere na osnove raspredeleniya mutnosti vody [A numerical study of impurity propagation in a freshwater lake on the basis of water turbidity distribution]. Computational Technologies. 22(1). pp. 113-124.

26. Tsydenov B.O. (2019) Simulating phytoplankton growth during the spring thermal bar in a deep lake. J. Mar. Syst. 195. P. 38-49. DOI: 10.1016/j.jmarsys.2019.03.009.

27. Shimaraev M.N., Verbolov V.I., Granin N.G., Sherstyankin P.P. (1994) Physical Limnology of Lake Baikal: A Review. Irkutsk; Okayama.

28. Votintsev K.K. (1978) Gidroximiya [Hydrochemistry]. Problemy 'Bajkala [The problems of Lake Baikal] (Galazii, G.I., Votintsev, K.K., Eds.). Novosibirsk. pp. 124-146.

29. Tsydenov B.O. (2018) Numerical modeling of the effect of inflow water mineralization in the dynamics of the autumnal thermal bar in Kamloops Lake. Moscow Univ. Phys. Bull. 73(4). pp. 435-440. DOI: 10.3103/S0027134918040148.

30. Katano T., Nakano S.-I., Ueno H., Mitamura O., Anbutsu K., Kihira M., Satoh Y., Satoh T., Drucker V.V., Tanaka Y., Akagashi Y., Sugiyama M. (2008) Abundance and composition of the summer phytoplankton community along a transect from the Barguzin River to the central basin of Lake Baikal. Limnology 9(3). pp. 243-250. DOI: 10.1007/s10201-008-0252-6.

31. Tsydenov B.O. (2018) Numerical modeling of the autumnal thermal bar. J. Mar. Syst. 179. pp. 1-9. DOI: 10.1016/j.jmarsys.2017.11.004.

32. Tsydenov B.O. (2019) Chislennoe modelirovanie vesenney dinamiki planktona na primere Selenginskogo melkovod'ya oz. Baykal [Numerical modeling of spring plankton dynamics in the Selenga shallow waters of Lake Baikal] Trudy KarNTs RAN [Trans. KarRC RAS] 3. pp. 114-123. DOI: 10.17076/lim948. 
33. Tomberg I.V., Sorokovikova L.M., Popovskaya G.I., Bashenkhaeva N.V., Sinyukovich V.N., Ivanov V.G. (2014) Concentration dynamics of biogenic elements and phytoplankton at Selenga R. Mouth and in Selenga shallows (Lake Baikal). Water Resources 41(6). pp. 687-695. DOI: $10.1134 / \mathrm{S} 0097807814050157$.

34. Domysheva V.M., Shimaraev M.N., Sakirko M.V., Onishhuk N.A. (2012) Dinamika koncentratsii biogennykh elementov i rastvorennykh gazov v vode ozera Baykal in our times [The dynamics of the concentration of nutrients and dissolved gases in water of Lake Baikal in the modern period] // Organicheskoe veshchestvo i biogennye elementy vo vnutrennikh vodoemakh i morskikh vodakh: Materialy V Vseross. simp. s mezhdunar. uchastiem (Petrozavodsk, 10-14 sent. 2012 g.) [Organic matter and biogenic elements in inland and marine waters: Proc. of the 5th all-Russian. Symp. with internat. participation]. Petrozavodsk. pp. 23-26.

Received: January 21, 2020 